\title{
OPIC, a kit for rapid merit function construction for use with all versions of OSLO, including OSLO EDU.
}

\author{
Brian Blandford*, Consultant, 5 Kingsway Mansions, Red Lion Square, London WC1R 4SE, UK.
}

The history of lens design software is sadly littered with accounts of excellent programs which fell by the wayside for lack of support. Others evolved through various package formats to form the foundation of today's very successful commercial software. One example of this is the Imperial College lens design program developed throughout the 1960s, 1970s and 1980s by Charles Wynne, Michael Kidger, Prudence Wormell, and others. This program (best known as the Kidger Optics Ltd SIGMA) produced many excellent designs over the years. One reason was that the ray patterns and weighting factors for operands in the default merit function had been carefully honed through experience, to produce rapid convergence on the global optimum from a likely starting point. This paper describes a suite of optimisation raysets and weighted operands written in the C-like OSLO compiled macro language CCL, and modeled on the Imperial College tradition. It is available for free download from http://www.lambdares.com/techsupport/kb/index.phtml. Its prime function is to provide a fast, easily understood introduction to merit function construction for the beginner. One version is for use on OSLO EDU, the free version of OSLO, which is also available from the Lambda Research Corporation website. This paper demonstrates how OPIC can be used to locate, from a remote starting point, the global minimum of the "monochromatic quartet," the lens design problem from the SPIE 1990 International Lens Design Conference.

KEYWORDS: OSLO, global minimum, merit function

\section{INTRODUCTION}

Ostensibly, this paper is about a shareware routine for lens optimisation, written in the C-like language CCL, to be embedded within a commercial lens design software package, and about the best form of merit function needed to locate the global minimum of a lens design problem. In fact, it also addresses a number of issues on the current shortage of lens designers, on how they are trained, and on the durability of lens design software.

\section{WHERE WILL THE NEXT GENERATION OF LENS DESIGNERS COME FROM?}

Every company which employs lens designers has the obligation to provide training and software. Software may be either home-grown, or commercial. As may be expected, home-grown programs are tailored to the specialised lens design requirements of the company which owns them, and much of the real cost of this software is hidden in the design budgets of individual projects, and in the long unpaid hours worked by the authors. Of course training lens designers to use these specialised packages does not necessarily qualify them to use other lens design software, a factor which might discourage career mobility. But the biggest disadvantage of specialised home-grown packages is that experience has proved that many do not survive long after the departure of the original authors. This may partly have been due to the failure of companies to address long-term software maintenance issues, but often it was the obsolescence of the operating system and hardware, which caused the loss of the software and the expertise embedded in it.

By contrast, as the popularity of in-house software has declined, commercial lens design programs have blossomed, supported by companies who have a vested interest in their survival, and who run comprehensive training courses in lens design, not only in the software. Many of the programs started as college teaching projects, and survived the transition from the educational to the commercial realm because of the active involvement of the original authors. OSLO ${ }^{\circ}$, originated by Doug Sinclair at the University of Rochester's Institute of Optics, is one example. It retains many features which were included for educational, rather than purely commercial reasons.

The unique capabilities of the free version, OSLO EDU, are a case in point. Most commercial optical software companies issue free demonstration versions of their products which are restricted to demonstrating the analysis 
capabilities, or with time limitations. They cannot be used for a serious design tasks in the long term. The main point of this paper is to demonstrate that the EDU version of OSLO, combined with the OPIC routine presented here, offers the potential to carry out serious design work on lens which fall within the 10 surface limit (including aperture stop and image). For the student, this means the opportunity to gain first-hand experience of completing a design task, up to and including the calculation of tolerances. This experience will be beneficial as training not only in the use of the paid-for versions of OSLO, but also in the use of other packages with facilities for damped least squares optimisation.

\section{WHERE ARE THE LENS DESIGN PROGRAMS OF YESTERYEAR?}

For many years, lens design software in the United Kingdom was dominated by a program written at Imperial College, London, originally by Prudence Wormell, under the supervision of Charles Wynne (reference 1). From its origins in FORTRAN, it was adapted by Michael Kidger and Davood Heshmaty Manesh to the Hewlett-Packard 9845, and later to the HP85. It provided for all the needs of a whole generation of lens designers in the UK. Because students trained at Imperial College were already proficient in its use, they could find ready employment in the factories where it had been adopted as the standard. It was sold by Imperial College with the source code, and this encouraged a valuable dialogue which helped identify and fix bugs, improve the documentation (which was already unsurpassed for its day), and permitted software fixes for particular lens design problems which could not be solved using the original program. Other versions were written, most notably one adapted for the zoom lens design at Rank Taylor Hobson in Leicester.

The successful commercialisation of this software was achieved by Michael and Tina Kidger with the establishment of Kidger Optics Ltd in 1982. The program was re-written in C language, and issued under the name SIGMA ${ }^{\circledR}$. To this day it retains many loyal users, despite the absence of updates. It retains all the best features of the Imperial College program, and incorporates many extensions such as tolerances and non-rotationally symmetric systems. The wealth of optical design knowledge incorporated in this program, and which formed the basis of Michael Kidger's training courses, are now available in two books of his, published posthumously (references 2,3).

It was an attempt to reproduce the best features of the optimisation merit function in this program, as well as a desire to get new lens designers quickly up to speed, that was the original motivation for the development of the OPIC routine.

\section{HOW DOES OPIC WORK?}

This section of the paper deals only with the EDU version of OPIC. Unlike the Standard and Premium versions, the EDU, Light, and old LT versions of OSLO make no provision for defining field point and ray sets. Also there are no pre-defined operands - all the quantities required are defined using a "callback routine," opcb_ic which evaluate the elements of the optimisation common matrix array, ocm[i]. This array is used to define the operand values in the operand spreadsheet, so that the merit function, otherwise known as the minimisation error function, $\psi$, is defined for most operands as

$$
\psi=\Sigma_{\mathrm{i}} \mathrm{W}_{\mathrm{i}}\left(\mathrm{ocm}_{\mathrm{i}}\right)^{2}
$$

where $\mathrm{W}_{\mathrm{i}}$ is the weighting factor for each operand. For a few operands (such as focal length or magnification, and overall lens length) the routine requires convergence on a non-zero value, called a target, and the term ocm in formula is then replaced by $\left(\mathrm{ocm}_{\mathrm{i}}-\operatorname{tgt}_{\mathrm{i}}\right)$.

For starting designs which suffer from vignetting of the off-axis beams, a call of the OPIC routine initiates a search for the top, bottom and side points of the pupil at both off-axis field positions. When the off-axis ray operands are evaluated in the callback routine, these vignetting values are used to define the off-axis ray sets. If the solution progresses to one where the vignetting has changed appreciably, another call to OPIC may be used to generate a new set of rays.

Because the number of operands in OSLO EDU is limited to 50, the chromatic variation of optical path difference ( $\delta \mathrm{OPD})$ is controlled, rather than the transverse ray aberrations at the second and third wavelengths. This variation is calculated using Conrady's formula

$$
\delta \mathrm{OPD}=\Sigma \delta \mathrm{n}(\mathrm{D}-\mathrm{d})
$$


where $\mathrm{D}$ is the path in glass of the ray in question, $\mathrm{d}$ the glass thickness along the axis, and $\delta$ n the dispersion. This is called the Conrady D minus d formula (reference 4, page 202) or DMD in OSLO nomenclature. Its use lies within the tradition of one of the options of the Imperial College and SIGMA programs, but it does not give satisfactory control of secondary spectrum. This is the reason why four chromatic Seidel terms and a finite transverse chromatic aberration have been added.

The sequence of activities initiated by a call to OPIC is as follows:

1. Search the pupil of the starting design (to an accuracy of 0.05 of pupil radius) for the two off-axis field points to find the vignetted values of the pupil rim coordinates $\mathrm{FY}_{\mathrm{MIN}}, \mathrm{FY}_{\mathrm{MAX}}, \mathrm{FX}_{\mathrm{MAX}}$. Store the values in text form on the 6 th line of the system note string array - a technique used in the GENII merit function supplied with OSLO. The points in the pupil illustrated in figure 1 are scaled according to the results of this pupil exploration exercise.

2. Set up optimisation conditions, and define opcb_ic as the optimisation call-back.

3. Define the operands, with non-zero targets as

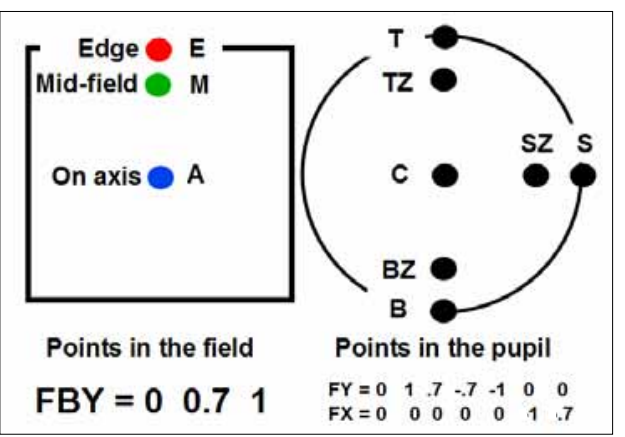

Figure 1 OPIC EDU ray set appropriate, and default weights for paraxial, Seidel (primary and secondary axial and lateral chromatic terms) and constructional parameters. Non-zero targets are calculated from the starting design, which is assumed to have the correct focal length or magnification.

4. Define the operands and default weights for finite axial rays at 1.0 and 0.7 relative pupil heights.

5. Define the operands associated with the 0.7 field pupil ray sagittal and tangential longitudinal paraxial focal shift, and distortion.

6. Define the operands for the rays in the vignetted pupil determined in stage 1 for the 0.7 field point.

7. Define the pupil ray operands for the edge of the field (as in 5, but adding the difference between the pupil ray heights for the 2 nd and $3 \mathrm{rd}$ wavelengths - i.e. the primary lateral colour).

8. Define the operands for the rays in the vignetted pupil for the edge of the field.

The ray patterns are chosen assuming rotational symmetry. For systems defined as focal, the monochromatic aberrations are transverse ray aberrations; for afocal systems they are angular ray aberrations.

One function of OPIC is to define the arbitrary reference strings listed in the

\begin{tabular}{|c|c|c|c|c|c|}
\hline \multicolumn{2}{|c|}{ SYSTEM TYPE } & $\mathbf{2 5}$ & M_B_DY & M_B_DYA \\
\hline No & FOCAL & AFOCAL & $\mathbf{2 6}$ & M_B_COL & M_B_COL \\
\hline $\mathbf{1}$ & PY & PY* & $\mathbf{2 7}$ & M_S_DX & M_S_DXA \\
\hline $\mathbf{2}$ & PU* & PU & $\mathbf{2 8}$ & M_S_DY & M_S_DYA \\
\hline $\mathbf{3}$ & PYC & PYC & $\mathbf{2 9}$ & M_S_COL & M_S_COL \\
\hline $\mathbf{4}$ & PUC & PUC & $\mathbf{3 0}$ & M_SZ_DX & M_SZ_DXA \\
\hline $\mathbf{5}$ & PAC & PAC & $\mathbf{3 1}$ & M_SZ_DY & M_SZ_DYA \\
\hline $\mathbf{6}$ & PLC & PLC & $\mathbf{3 2}$ & M_SZ_COL & M_SZ_COL \\
\hline $\mathbf{7}$ & SAC & SAC & $\mathbf{3 3}$ & E__FS & E_XFSA \\
\hline $\mathbf{8}$ & SLC & SLC & $\mathbf{3 4}$ & E_YFS & E_YFSA \\
\hline $\mathbf{9}$ & OALL* & OALL* & $\mathbf{3 5}$ & E_DIST\% & E_DIST\% \\
\hline $\mathbf{1 0}$ & EFL* & AMAG* & $\mathbf{3 6}$ & E_PLC & E_PLCA \\
\hline $\mathbf{1 1}$ & [USER] & [USER] & $\mathbf{3 7}$ & E_T_DY & E_T_DYA \\
\hline $\mathbf{1 2}$ & A_DY & A_DYA & $\mathbf{3 8}$ & E_T_COL & E_T_COL \\
\hline $\mathbf{1 3}$ & A_COL & A_COL & $\mathbf{3 9}$ & E_TZ_DY & E_TZ_DYA \\
\hline $\mathbf{1 4}$ & A_Z_DY & A_Z_DYA & $\mathbf{4 0}$ & E_TZ_COL & E_TZ_COL \\
\hline $\mathbf{1 5}$ & A_Z_COL & A_Z_COL & $\mathbf{4 1}$ & E_BZ_DY & E_BZ_DYA \\
\hline $\mathbf{1 6}$ & M_XFS & M_XFSA & $\mathbf{4 2}$ & E_BZ_COL & E_BZ_COL \\
\hline $\mathbf{1 7}$ & M_YFS & M_YFSA & $\mathbf{4 3}$ & E_B_DY & E_B_DYA \\
\hline $\mathbf{1 8}$ & M_DIST\% & M_DIST\% & $\mathbf{4 4}$ & E_B_COL & E_B_COL \\
\hline $\mathbf{1 9}$ & M_T_DY & M_T_DYA & $\mathbf{4 5}$ & E_S_DX & E_S_DXA \\
\hline $\mathbf{2 0}$ & M_T_COL & M_T_COL & $\mathbf{4 6}$ & E_S_DY & E_S_DYA \\
\hline $\mathbf{2 1}$ & M_TZ_DY & M_TZ_DYA & $\mathbf{4 7}$ & E_S_COL & E_S_COL \\
\hline $\mathbf{2 2}$ & M_TZ_COL & M_TZ_COL & $\mathbf{4 8}$ & E_SZ_DX & E_SZ_DXA \\
\hline $\mathbf{2 3}$ & M_BZ_DY & M_BZ_DYA & $\mathbf{4 9}$ & E_SZ_DY & E_SZ_DYA \\
\hline $\mathbf{2 4}$ & M_BZ_COL & M_BZ_COL & $\mathbf{5 0}$ & E_SZ_COL & E_SZ_COL \\
\hline
\end{tabular}

\section{Table 1 Mnemonic labels for OPIC EDU operands} operand column labeled "Name". These enable finite ray operands to be easily identified, using simple mnemonics as illustrated in figure 1 . For example, the $\mathrm{X}$ component of the angular aberration of the ray in the "zone" of the pupil sagittal section $(\mathrm{FY}=0.0, \mathrm{FX}=0.7)$, for the edge-of-field object point $(\mathrm{FBY}=1.0)$ is named "E_SZ_DXA". The names are listed in table 1 for systems with both focal and afocal image space assessment. Asterisks refer to operands set with non-zero targets.

Once the operand set is defined, it needs to be checked for realism. The targets for the parameters derived from the starting design may be inaccurate, and weighting factors may need adjustment. In particular the colour weights may all 
be too high or too low relative to the monochromatic aberrations. Provision has been made for the call of OPIC to be followed by a numerical colour weight $($ default $=1)$ which will scale them all in the same ratio - e.g. opic $\mathbf{3 . 0}$

Once the merit function has been checked, and a set of variables has been defined, optimisation commences with a call to the damped least squares routine or (less frequently) the Nelder and Mead simplex method, an optimisation technique which works by direct search, rather than gradients. For each evaluation of the merit function there is a call to the callback routine, opcb ic, which:

1. Scrolls the spreadsheet buffer to preserve the current contents of the text window.

2. Extracts the numeric values of the coordinates of the off-axis pupils from the text of the system note.

3. Evaluates all the operand values, and assigns them to consistent array terms within the spreadsheet

buffer $\operatorname{ssb}()$.

4. Assigns elements of the ocm[] array to the corresponding spreadsheet buffer elements.

5. Scrolls back the spreadsheet buffer to restore the appearance of the associated text window. Optimisation will proceed until the percentage fall in the merit function is less than $10^{-6}$.

\section{CAN OPIC LOCATE THE GLOBAL MINIMUM OF A LENS DESIGN PROBLEM?}

Because most problems in lens design have many hundreds of more-or-less equivalent solutions, it is hard to know exactly when a global optimum has been found. There is perhaps one occasion when this has been achieved. A problem was devised by David Shafer which was sufficiently simple, and which was attempted by a sufficiently large number of designers, that we can safely assume that the winner was indeed the best achievable.

The task was specified as follows: "Design a four element $\mathrm{f} / 3$ objective of $100 \mathrm{~mm}$ equivalent focal length, made only of $B K 7$ to be used at the 0.58756 micron (helium d) wavelength $(n=1.51680)$. The object is at infinity, the field angle is 30 degrees (15 degrees semi-field), the image is flat. The minimum glass thickness is $2 \mathrm{~mm}$, the distortion must be less than $1 \%$, and there can be no vignetting. The entries will be judged on the mean RMS blur circle diameter at the three field points, on axis, 10.5 degrees and 15 degrees, equally weighted."

Designs which had the last surface either crossing or after the image plane, were disqualified (i.e. the image must be real and in air). Designs which had the stop in glass were accepted - it is feasible to manufacture by turning a groove in the edge of a thick lens. There is no minimum air gap - solutions which permitted lenses to touch at the edge were acceptable.

The results of the competition were presented by Don O'Shea (reference 5). Forty four solutions were submitted, and the best three had practically the same appearance and performance. It was David Williamson who submitted the best design, illustrated in figure 2, which has a mean RMS spot size of $2.4 \mu \mathrm{m}$. The prescription for this is:

\begin{tabular}{|c|c|c|c|c|}
\hline \multicolumn{5}{|c|}{ Monochromatic quartet winner \#14 } \\
\hline SRF & RADIUS & THICKNESS & APERTURE RADIUS & GLASS \\
\hline OBJ & - - & $1.0000 \mathrm{e}+20$ & $2.6795 e+19$ & AIR \\
\hline 1 & 145.687500 & V $\quad 2.000000$ & V 65.000000 & BK7 \\
\hline 2 & 94.711600 & 10.803900 & 62.000000 & AIR \\
\hline 3 & 162.727400 & 231.497100 & 62.000000 & BK7 C \\
\hline 4 & -143.653900 & $--\quad v$ & $13.514396 \mathrm{~s}$ & AIR \\
\hline AST & - - & $0.100000 \mathrm{~V}$ & $13.567000 \mathrm{~A}$ & AIR \\
\hline 6 & 68.400400 & 103.229400 & $13.540343 \mathrm{~S}$ & BK7 \\
\hline 7 & $-1.4803 e+03$ & 6.721500 & 23.500000 & AIR \\
\hline 8 & -43.033700 & 2.000000 & 23.000000 & BK7 C \\
\hline 9 & 858.342000 & 2.003810 & V 26.000000 & AIR \\
\hline IMS & - - & -- & $26.767792 \mathrm{~S}$ & * \\
\hline
\end{tabular}

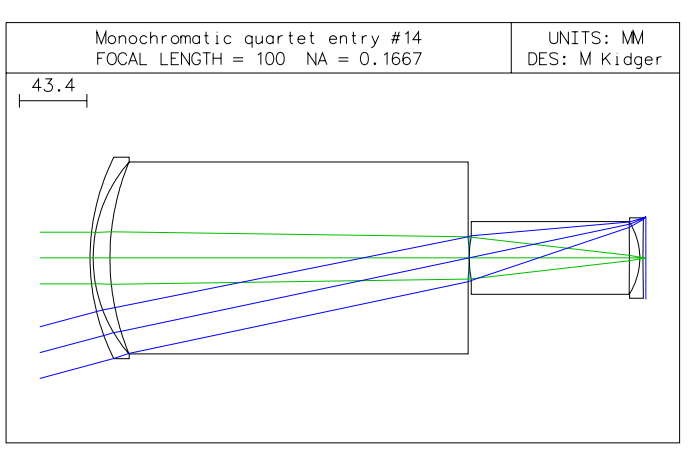

Figure 2 The winning design

The exercise which will now be described is to devise a credible starting design for a lens to meet this specification, and then, using the OPIC error function with slight modification, to try to attain, as close as possible, the global optimum solution described above. 
The starting design, which is illustrated in figure 3, consists of a front negative lens (in common with many wide-angle systems), two plano-convex lenses with the aperture stop placed symmetrically between them, and finally a field flattening lens near the image.

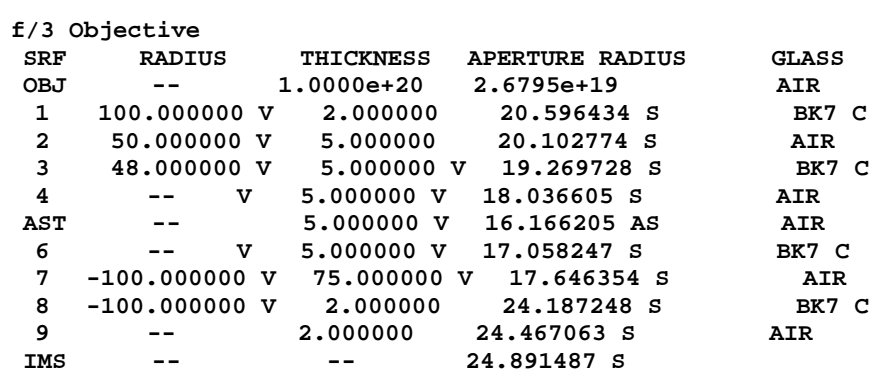

For the first optimisation cycles, all the curvatures, air spaces, and glass thicknesses are allowed to vary with the exception of the last

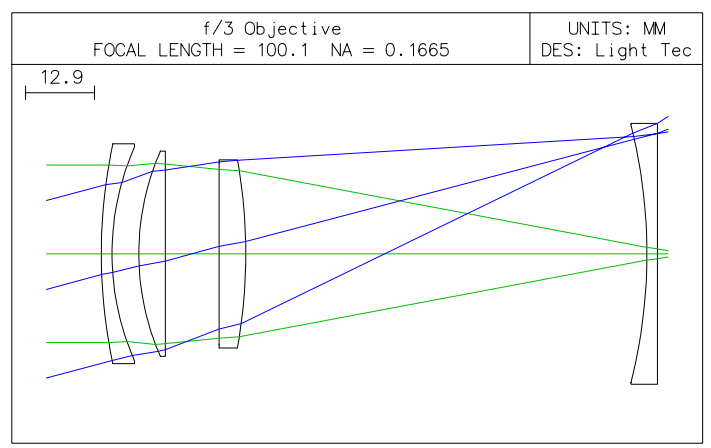

Figure 3 The starting design curvature, the first and last glass thickness, and the first and last airspaces. A $2 \mathrm{~mm}$ minimum is placed on all glass thicknesses.

After the call to OPIC, and the operand spreadsheet is opened (ose), and the weight on overall lens length (OALL) is

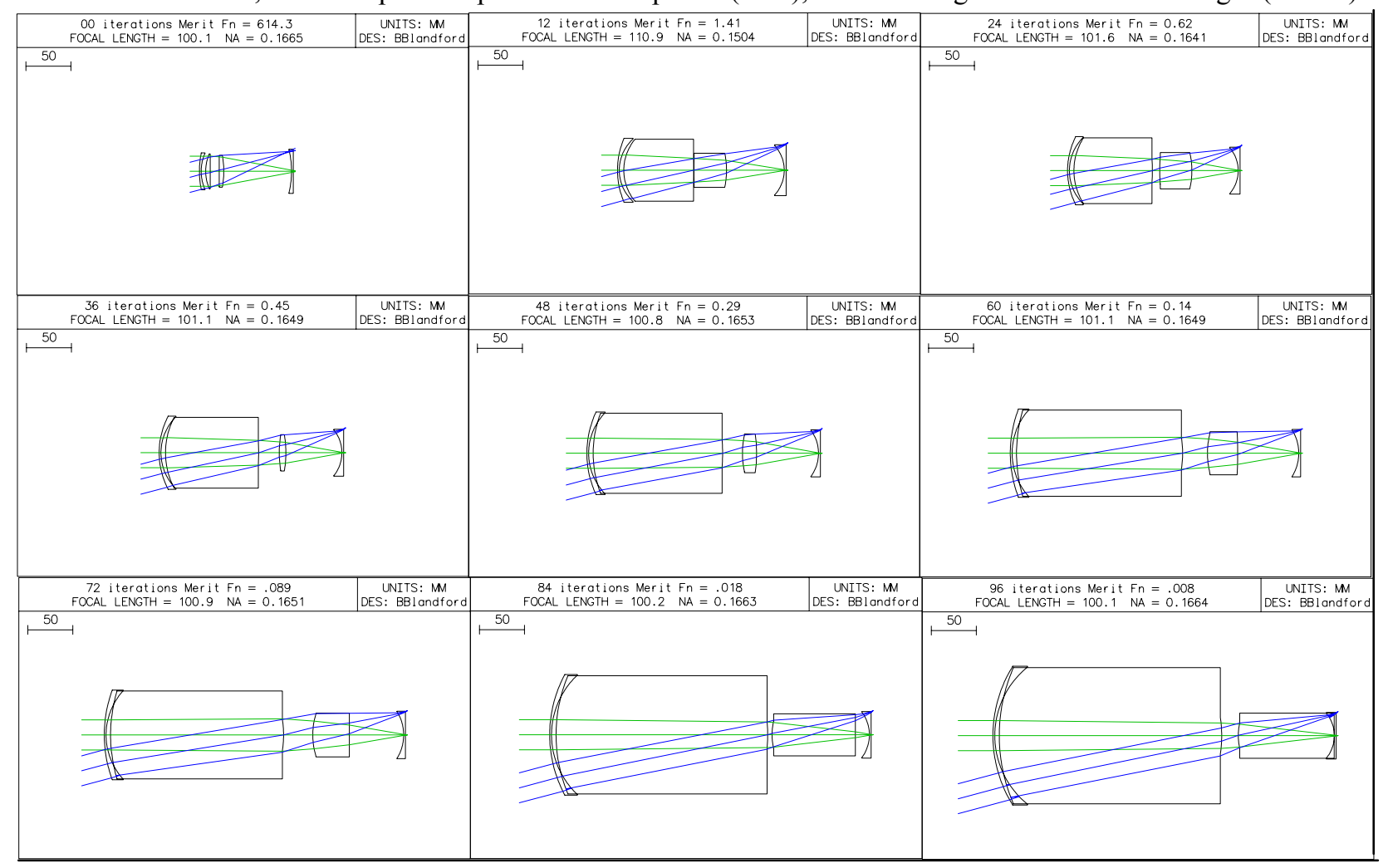

Figure 4 The sequence of solutions using OPIC EDU with standard ray set

set to zero (it is not a constraint in the competition). The weight on percent distortion at the edge of the field (E_DIST\%) is increased to 0.01 . Of course the colour weights are all zero as only one wavelength is defined. Optimisation then proceeds with a call of ite ful 12, the OSLO command for 12 cycles of damped least squares optimisation. The progress of the design through parameter space, which can be verified by the reader, is illustrated in the sequence of 
diagrams in figure 4. After 36 iterations there is a close encounter with a local minimum, in which the third lens becomes so thin that it approaches the $2 \mathrm{~mm}$ limit. After 12 more iterations, however, the lens has begun to grow again, until it eventually fills the entire space between the stop and the last lens.

After 96 iterations, the design has clearly moved into the region of the global optimum shown in figure 2, and the merit function is no longer falling. However, the last image in figure 4 indicates that the edge thicknesses of two air spaces have gone negative, and this needs to be rectified.

Automatic control of edge and centre thicknesses is a standard feature in OSLO Standard and Premium, but in the case of OSLO EDU the preferred way route is by means of slider wheel optimisation. Adding the last curvature (9) to the list of variables, the two offending air spaces $(2,7)$ are adjusted by hand using "slider wheel callback" - an OSLO feature which permits up to 10 cycles of optimisation for each increment of either parameter. This gives the design shown in figure 5. The mean RMS spot size for this interim solution is $2.6 \mu \mathrm{m}$, which already puts this design in the top tier of solutions described in reference 5 .

Only 49 operands have been used in the optimisation exercise so far. The last operand is available for user-defined quantities - anything that can be printed in the spreadsheet buffer can be assigned to the relevant matrix element ocm[11]. Although it is intended that this part of the OPIC routine should be modified by a user proficient in CCL programming, the standard version in fact already has the desired parameter, the sum of the RMS spot sizes at three field points $(0,0.7,1.0)$, as the default operand. It only remains, therefore, to set the weight on operand 11 to $10^{4}$ and re-optimise. After increasing the weighting on the image space paraxial marginal ray angle PU (which controls the focal length) from $10^{3}$ to $10^{6}$, and a further adjustment of the front air space (2), the design shown in figure 6 is obtained. This has a mean RMS spot size of $2.4 \mu \mathrm{m}$, which is the same as the published value of the winning solution. The prescription for this is shown below:

$\begin{array}{ccccc}\begin{array}{c}\text { Mean } \\ \text { SRF }\end{array} & \text { RMS Spot size } & \text { optimised } & & \\ \text { RADIUS } & \begin{array}{c}\text { THICKNESS } \\ \text { APERTURE RADIUS }\end{array} & \text { GLASS } \\ \text { OBJ } & -- & 1.0000 \mathrm{e}+20 & 2.6795 \mathrm{e}+19 & \mathrm{~A} \\ 1 & 133.989472 \mathrm{~V} & 2.000000 & 70.181266 \mathrm{~S} & \text { BK7 C } \\ 2 & 93.804450 \mathrm{~V} & 12.600000 & 69.471034 \mathrm{~S} & \text { AIR } \\ 3 & 183.844301 \mathrm{~V} & 234.874126 \mathrm{~V} & 67.506687 \mathrm{~S} & \text { BK7 C } \\ 4 & -143.067485 \mathrm{~V} & 0.712035 \mathrm{~V} & 13.980702 \mathrm{~S} & \text { AIR } \\ \text { AST } & -- & 0.205066 \mathrm{~V} & 13.698616 \mathrm{AS} & \text { AIR } \\ 6 & 68.791456 \mathrm{~V} & 104.912555 \mathrm{~V} & 13.751080 \mathrm{~S} & \text { BK7 C } \\ 7 & -1.3257 \mathrm{e}+03 \mathrm{~V} & 6.636409 & 24.301498 \mathrm{~S} & \text { AIR } \\ 8 & -43.099770 \mathrm{~V} & 2.000000 & 25.250917 \mathrm{~S} & \text { BK7 C } \\ 9 & 1.0738 \mathrm{e}+03 \mathrm{~V} & 2.000000 & 25.838787 \mathrm{~S} & \text { AIR } \\ \text { IMS } & -- & -- & 26.755339 \mathrm{~S} & \end{array}$

It may not be thought remarkable that this solution has been found using a starting design and merit function similar to that used for the original winning design. What is remarkable, however, is that this global minimum has been found using free software, with a limit of 50 operands.

The success of the augmented merit function in locating the global minimum begs the question as to why this same merit function was not used from the start. The reason is that if it is applied to the starting design, the design converges rapidly on a local minimum, with a mean RMS spot size of $30 \mu \mathrm{m}$, illustrated in figure 7 .

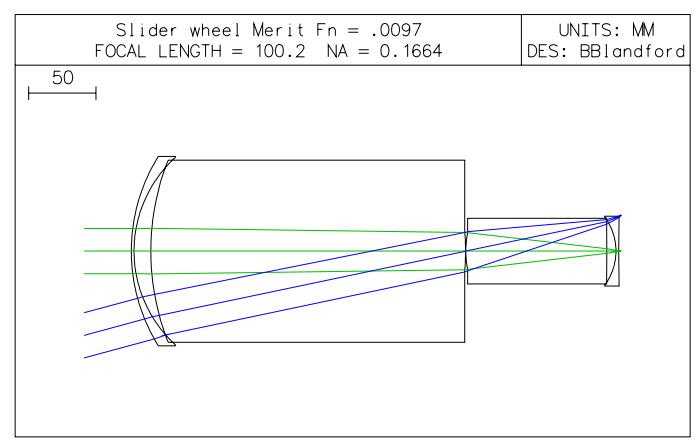

Figure 5 After adjustment of air spaces

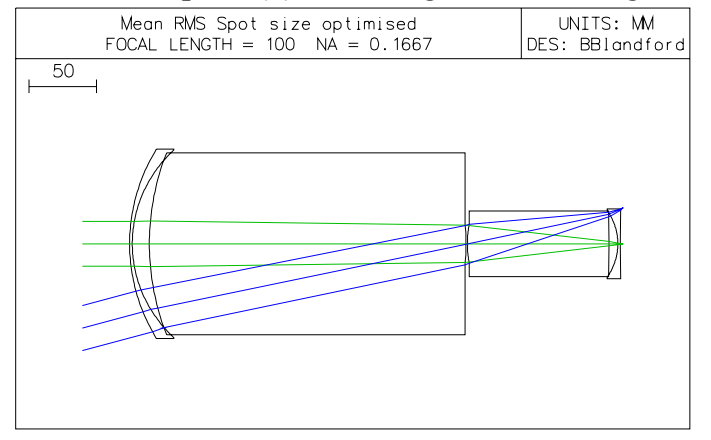

Figure 6 After dense ray set optimisation

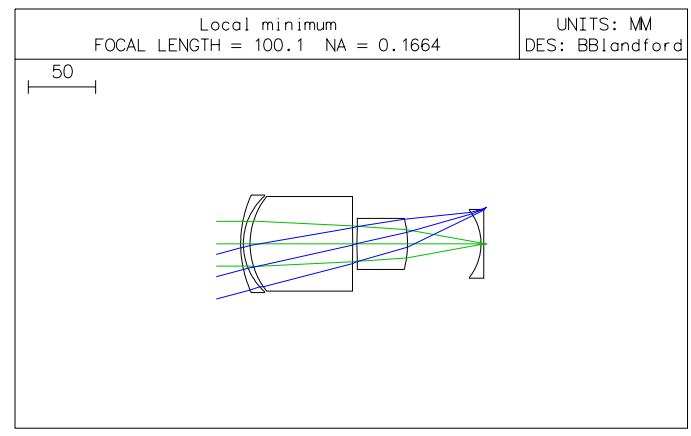

Figure 7 A non-optimum solution 
This emphasises an important aspect of the Imperial College approach - weights are to be regarded as not static but dynamic. The number of rays in the optimisation was at the time limited by the speed and capacity of the hardware. However experience has shown that looking for the "valley" of the global minimum from a remote starting point requires the designer to start exploration with a sparse ray set and light initial control of focal length and edge thicknesses. Only when the "valley" has been found should the number of rays be increased, and the Gaussian and constructional parameters finely adjusted to home in on the best solution. David Williamson has confirmed that he started his winning design with a negative-positive-positive-negative starting point, using SIGMA with its (then) limited ray set to obtain an interim design similar to that shown in figure 5. He then completed the design using a dense ray set on CODE $V ®$. He comments, however, that since the inclusion of so-called global optimisation routines in the more comprehensive versions of all the main commercial packages, even four parallel plates can now be used as a starting point for finding the optimum of this particular problem. And it is his original solution that is found.

\section{WHERE DO WE GO FROM HERE?}

Versions of OPIC for the Standard and Premium editions of OSLO have been presented by the author as a feature of OSLO training courses in recent years. Because operands can be defined directly, rather than through the ocm[i] array, and because the ray coordinates in the pupil can be adjusted manually to take account of vignetting, these routines are easier to use. Colour correction is done with operands calculated at three different wavelengths, weighted according to the spectral weightings in the starting design wavelength spread sheet. There are also different versions for lenses with high apertures, with wide fields of view, etc. It is intended that these too will shortly be available from the Lambda Research Corporation website, together with full documentation. In particular the OPIC EDU instructions are written at a level where someone with no experience in lens design whatever can quickly get a real flavour of the subject.

This prompts a possibly controversial solution to the current shortage of lens designers discussed in section 2 , which is that lens design might be done in future as a part-time activity. The lens designer will have another occupation as a "day job," such as mechanical designer, electronic designer or systems engineer. There are two reasons why this is possible: firstly, today's powerful combination of software and hardware speeds up and simplifies the lens design process considerably; and secondly, one of the most time consuming aspects of lens design is the management of the interface with other engineering disciplines, so that combining two professions can only be beneficial. Whether or not this happens, I hope at least that this exercise will stimulate the interest of a few newcomers to look further into this fascinating subject.

\section{ACKNOWLEDGEMENTS}

The assistance of Chun-Hong Chen, Panos Eracleous, Alison Fairhurst, Tina Kidger, Jon Maxwell, David Williamson, and Prudence Wormell in the preparation of this paper is gratefully acknowledged. Jon Maxwell points out that Opic was the trade name chosen by Taylor, Taylor and Hobson for Horace W. Lee's f/2 masterpiece of 1921, which later became the basis of the Speed Panchro series of cine lenses (see reference 6).

OSLO is a registered trademark of Lambda Research Corporation, CODE V is a registered trademark of Optical Research Associates. The copyright of SIGMA is the property of Zemax Development Corporation.

\section{REFERENCES}

1. CG Wynne, PMJH Wormell, "Lens Design by Computer” Applied Optics, Vol 2, pp.1233-1238, 1963.

2. Michael J. Kidger, Fundamental Optical Design, SPIE Press, 2001.

3. Michael J. Kidger, Intermediate Optical Design, SPIE Press, 2004.

4. Walter T Welford, Aberrations of Optical Systems, Adam Hilger Ltd, 1986.

5. Donald C. O'Shea, "Monochromatic quartet: a search for the global optimum" 1990 International Lens Design Conference; George N. Lawrence; Ed. Proc. SPIE 1354, pp 548-554, 1991.

6. Rudolph Kingslake, A History of the Photographic Lens, Academic Press, 1989. 
*Email: brian.blandford@physics.org 